\title{
Caseous Calcification of the Mitral Annulus: From Asymptomatic Lesion to Embolic Complication- Case Report
}

\author{
Slavica Mitrovska, MD, PhD ${ }^{1 *}$, Sasko Jovev², Vasil Papestiev² and Vesna Mitashova ${ }^{2}$ \\ ${ }^{1}$ Department of Internal Medicine, Promedika Medical Centre, Skopje, Macedonia \\ ${ }^{2}$ Department of Cardiac Surgery, University Clinic for State Cardiac Surgery, Skopje, Macedonia
}

*Corresponding author: Slavica Mitrovska, MD, PhD, Department of Internal Medicine, Promedika Medical Centre, Blv. Cyril and Methodius No.50, 1000 Skopje, Republic of Macedonia, Tel: 0038971385267

\section{Introduction}

Caseous calcification of the mitral annulus (CCMA) is a rare form of mitral annular calcification with a prevalence of $0.06 \%$ in the general population [1]. It is considered a benign condition, but in some cases it carries a risk for cardiovascular and cerebrovascular events. There is no consensus regarding treatment strategy and surgery is recommended for mitral valve dysfunction or thromboembolic events [2]. We present a case of surgical management of caseous mass of the posterior commissures of the mitral annulus with rapid dynamics from asymptomatic lesion to embolic complication.

\section{Case Description}

In October 2016, a 55-year-old female patient with a history of arterial hypertension and dyslipidemia presented to our department for a regular checkup. She had body mass index of $35.8 \mathrm{~kg} / \mathrm{m}^{2}$, blood pressure of $190 / 110 \mathrm{mmHg}$ and regular pulse of $82 \mathrm{bpm}$. Auscultatory findings were unremarkable. Laboratory analysis identified total cholesterol of 8.2 $\mathrm{mmol} / \mathrm{I}$ and LDL-c of $4.2 \mathrm{mmol} / \mathrm{l}$. The ECG showed sinus rhythm, no significant abnormalities. A transthoracic echocardiogram (TTE) revealed a spherical hyperechoic mass with a central hypoechogenic lesion on the posterior subvalvular angle, $34 \times 19 \times 23 \mathrm{~mm}$ in size, smooth borders and no acoustic shadow artifacts. Additional findings were mild mitral regurgitation, mild left atrium dilatation, and preserved ejection fraction (EF) of 56\% (Figure 1A). To make a definitive diagnosis cardiac magnetic resonance imaging (CMR) was recommended. It confirmed well defined oval mass with a bright signal on $\mathrm{T} 1$, low-intensity signal on T2 (peripheral calcification with central liquid core) and no late gadolinium enhancement (LGE) (Figure 1B).

In February 2017, the patient complained of palpitations, dyspnea on exertion and cough over the past month. ECG revealed sinus tachycardia and diffuse nonspecific ST changes. TTE showed a slight increase in lesion size $(36 \times 22 \times 24 \mathrm{~mm})$, dilatation of the left ventricle with borderline $\mathrm{EF}=50 \%$. There was also mild mitral stenosis with mild mitral regurgitation. Coronary angiography revealed normal coronary arteries (Figure 1C).

These signs and symptoms put the patient at risk of complications and surgery was considered. Patient declined the surgery and was sent home with a scheduled follow-up for 3 months. She came a year later (January 2018), because of worsening dyspnea and palpitations for 1 month. TEE confirmed large mitral annular calcification measuring $44 \times 28 \times 32$ (Figure 1D). Surgical treatment was refused again.

In November 2018, the patient described an episode of chest pain, shortness of breath, cough, and bloody sputum a month earlier. This data raised our suspicions of a possible pulmonary embolism. TTE showed progression to moderate mitral regurgitation, with no evidence of infective endocarditis. Finally, we received written consent for surgery.

Citation: Mitrovska S, Jovev S, Papestiev V, Mitashova V (2022) Caseous Calcification of the Mitral Annulus: From Asymptomatic Lesion to Embolic Complication- Case Report. Int J Clin Cardiol 9:247. doi.org/10.23937/2378-2951/1410247

Accepted: January 22, 2022: Published: January 24, 2022

Copyright: (C) 2022 Mitrovska S, et al. This is an open-access article distributed under the terms of the Creative Commons Attribution License, which permits unrestricted use, distribution, and reproduction in any medium, provided the original author and source are credited. 

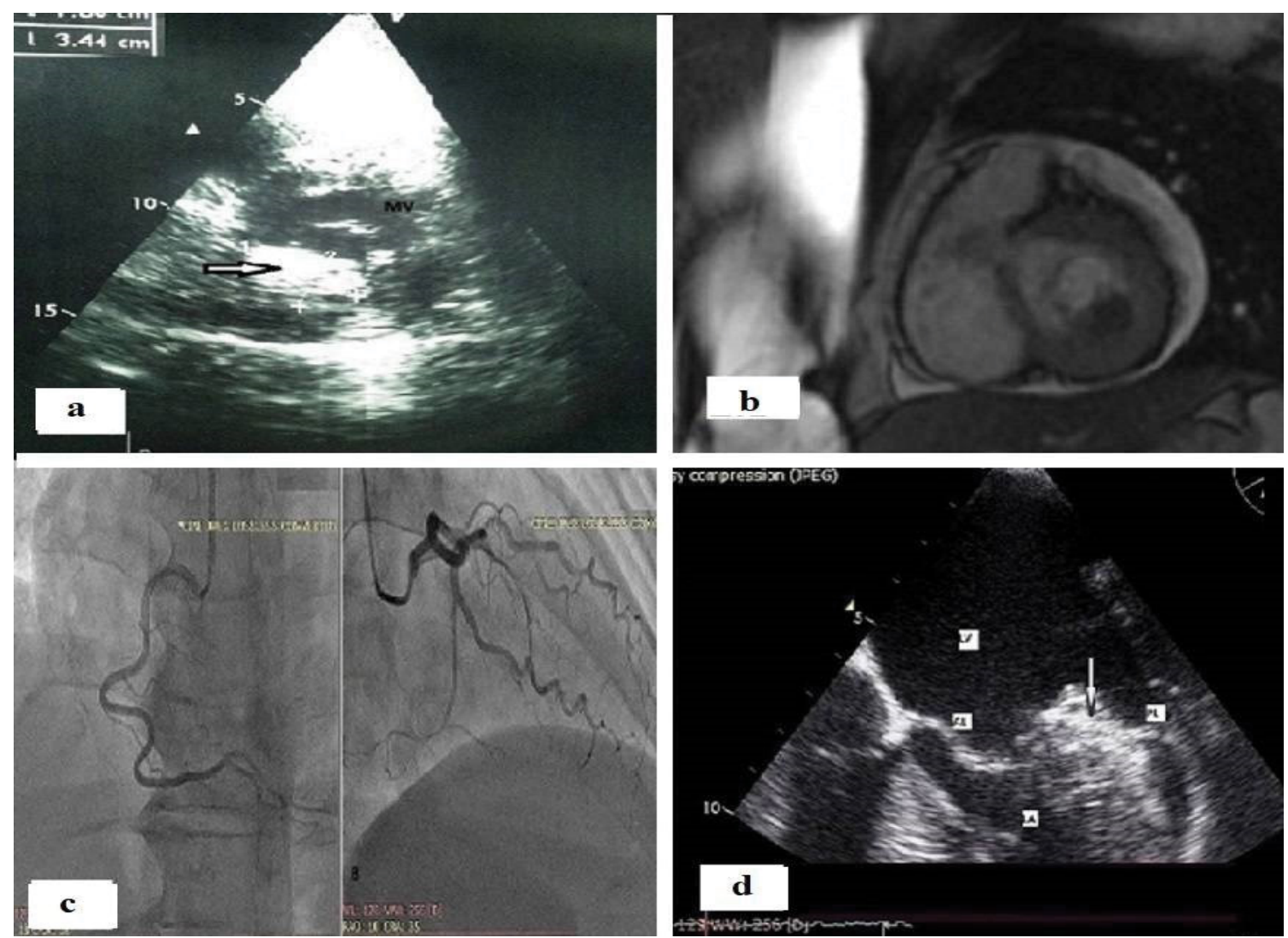

Figure 1: a) Transthoracic echocardiogram: Short axis views: A spherical hyperechoic mass with central hypoechogenic lesion (arrow), MV: Mitral Valve; b) Cardiac magnetic resonance: sessile, calcified amorphous mass at the base of the posterior leaflet of the mitral valve; c) Selective coronary angiography: Normal right, left anterior descending and circumflex artery; d) Transesophageal echocardiography: midesophageal four-chamber view: large mitral annular calcification (arrow).

AL: Anterior Leaflet; PL: Posterior Leaflet, LA: Left Atrium; LV: Left Ventricle
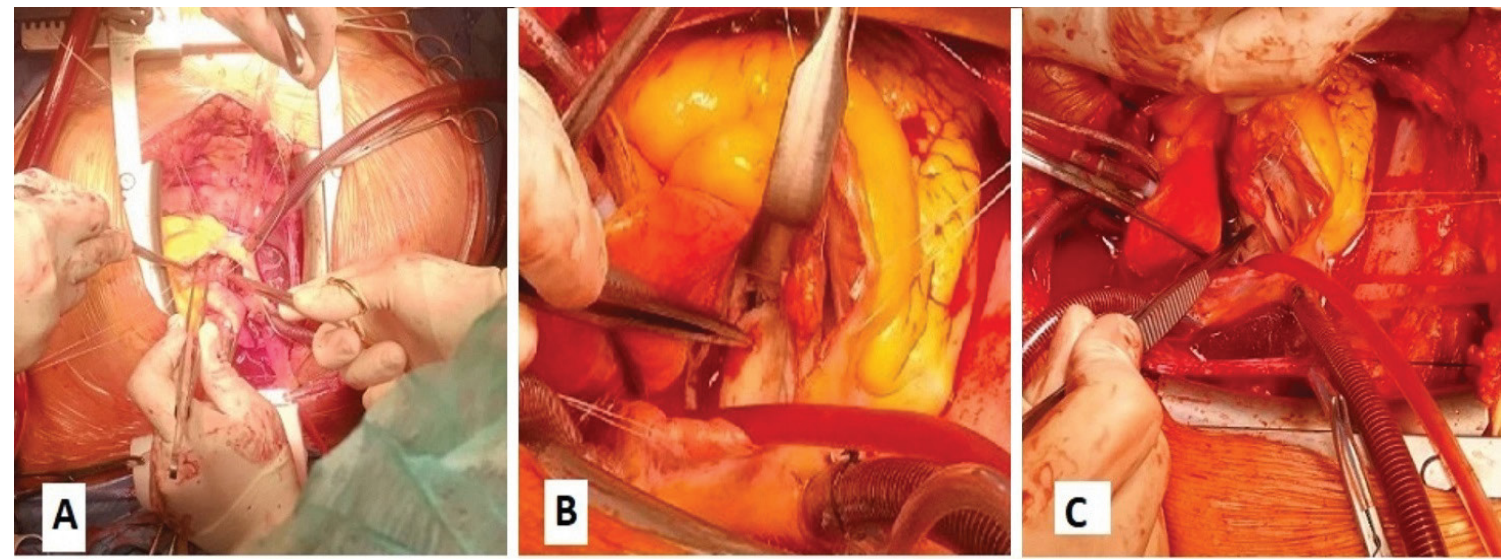

Figure 2: Intraoperative findings: A) and B) Excision of the posterior mitral annulus; C) Pericardial patch closure.

On-pump cardiopulmonary bypass was performed under general anaesthesia and median sternotomy. Myocardial protection was achieved using antegrade and retrograde cold cardioplegia. During the cardiotomy, a mass incision was performed and gelatinous material flowed out. This was followed by complete excision and reconstruction of the posterior mitral annulus with the pericardial patch (Figure 2).

Weaning from the cardiopulmonary bypass was unremarkable. Histological analysis showed amorphous, eosinophilic material mixed with inflammatory cells (plasma cells and lymphocytes). No malignant or myxoma cells were found. The two-year follow-up period was without any adverse events.

\section{Discussion}

Caseous mitral calcification is a clinically silent but it is thought to be an active process similar to atherosclerosis. The presence of risk factors-hypertension, dyslipidemia, and diabetes mellitus contribute to endothelial dysfunction, activation of signaling pathways, and releasing of inflammatory cytokines. They lead to the 
valve structural modification by collagen accumulation, fibrosis and tissue stiffness. The morphological feature is composed of peripheral calcification and lipid core and can be detected by transthoracic echocardiography [3]. But additional imaging modalities are required to avoid misinterpretation such as an abscess or tumor. Thus, Mallat N, et al. in their study described the lesion as liquefactive necrosis often associated with bacterial, viral or fungal infection [4]. Chen 0 , et al. concluded that $C M R$ is an excellent imaging modality because of tissue characterization [5]. We presented a case of a caseous mass incidentally found on TTE and confirmed with CMR as a sessile, calcified amorphous mass with the bright signal on $\mathrm{T} 1$, low-intensity signal on $\mathrm{T} 2$, and no LGE enhancement.

Caseous mass can increase in size and it is associated with adverse events. In Framingham Heart Study, for every $1-\mathrm{mm}$ increase in mitral annular calcification, the risk of incident CVD, death, and all-cause mortality increased by $9 \%(p<0.007), 12 \%(p<0.004)$ and $9 \%$ ( $p$ $<0.001$ ) [6]. Sagnier S, et al. described a patient with CCMA associated with stroke [7].

Our case is an illustration of the rapid growth of a CCMA with progression from asymptomatic to symptomatic lesion, highly suspected of being associated with pulmonary embolism. This case was also a therapeutic challenge. Due to the patient's noncompliance with our recommendations, the operation was performed lately, after the embolic complication. Fortunately, there are no adverse events in the twoyear postoperative period.

\section{Conclusions}

Our case concludes that pulmonary embolism is a possible clinical scenario in patients with CCMA. The study emphasized the importance of a comprehensive diagnostic approach to establish an accurate diagnosis and identify high-risk patients who may benefit from surgical treatment.

\section{Conflict of Interest}

The authors certify that there is no conflict of interest with any financial organization regarding the material discussed in the manuscript.

\section{Statement of Equal Author's Contribution}

All authors made equal contribution.

\section{Funding}

None reported.

\section{References}

1. Pomeroy LW, Markelz B, Steel K, Slim MA (2013) Mitral annular caseous calcification: A rare variant of a common echocardiographic finding discovered with advanced imaging techniques. Case Rep Med 2013: 972684.

2. Kanjanauthai S, Nasir K, Katz R, Rivera JJ, Takasu J, et al. (2010) Relationships of mitral annular calcification to cardiovascular risk factors: The Multi-Ethnic Study of Atherosclerosis (MESA). Atherosclerosis 213: 558-562.

3. Calafiore AM, Totaro A, Testa N, Sacra C, Castellano G, et al. (2021) The secret life of the mitral valve. J Card Surg 36: $247-259$

4. Mallat N, Limeme M, Zaghouani H, Mootemri F, Majdoub S, et al. (2013) Caseous calcification of the mitral annulus on MDCT: A rare intracardiac mass. Acta Radiol Short Rep 2: 2047981613502177.

5. Chen O, Dontineni N, Nahlawi G, Bhumireddy GP, Han SY, et al. (2012) Serial cardiac magnetic resonance imaging of a rapidly progressing liquefaction necrosis of mitral annulus calcification associated with embolic stroke. Circulation 125: 2792-2795.

6. Fox CS, Vasan RS, Parise H, Levy D, O'Donnell CJ, et al. (2003) Mitral annular calcification predicts cardiovascular morbidity and mortality: The Framingham Heart Study. Circulation 107: 1492-1496.

7. Sagnier S, Poli $M$, Oysel-Mestre $M$, Corneloup $O$, Debruxelles S, et al. (2015) Caseous calcification of the mitral annulus associated with stroke: Report of two cases. Rev Neurol 171: 157-160. 